\title{
Efficient reaction deletion algorithms for redesign of constraint-based metabolic networks for metabolite production with weak coupling
}

Takeyuki Tamura ( $\square$ tamura@kuicr.kyoto-u.ac.jp )

Kyoto University

\section{Research article}

Keywords: metabolic network, ux balance analysis, growth coupling, linear programming, algorithm, constraint-based model

Posted Date: August 5th, 2020

DOI: https://doi.org/10.21203/rs.2.24108/v2

License: (c) (1) This work is licensed under a Creative Commons Attribution 4.0 International License. Read Full License

Version of Record: A version of this preprint was published at IPSJ Transactions on Bioinformatics on January 1st, 2021. See the published version at https://doi.org/10.2197/ipsjtbio.14.12. 


\section{RESEARCH}

\section{Efficient reaction deletion algorithms for redesign of constraint-based metabolic networks for metabolite production with weak coupling}

Takeyuki Tamura

\author{
Correspondence: \\ tamura@kuicr.kyoto-u.ac.jp \\ Bioinformatics Center, Institute \\ for Chemical Research, Kyoto \\ University, Gokasho, Uji, Japan \\ Full list of author information is \\ available at the end of the article
}

\begin{abstract}
Background: Metabolic engineering strategies enabling the production of specific target metabolites by host strains can be identified in silico through the use of metabolic network analysis such as flux balance analysis. This type of metabolic redesign is based on the computation of reactions that should be deleted from the original network representing the metabolism of the host strain to enable the production of the target metabolites while still ensuring its growth (the concept of growth coupling). In this context, it is important to use algorithms that enable this growth-coupled reaction deletions identification for any metabolic network topologies and any potential target metabolites. A recent method using a strong growth coupling assumption has been shown to be able to identify such computational redesign for nearly all metabolites included in the genome-scale metabolic models of Escherichia coli and Saccharomyces cerevisiae when cultivated under aerobic conditions. However, this approach enables the computational redesign of $S$. cerevisiae for only $3.9 \%$ of all metabolites if under anaerobic conditions. Therefore, it is necessary to develop algorithms able to perform for various culture conditions.

Results: The author developed an algorithm that could calculate the reaction deletions that achieve the coupling of growth and production for $91.3 \%$ metabolites in genome-scale models of $S$. cerevisiae under anaerobic conditions. Computational experiments showed that the proposed algorithm is efficient also for aerobic conditions and Escherichia coli. In these analyses, the least target production rates were evaluated using flux variability analysis when multiple fluxes yield the highest growth rate. To demonstrate the feasibility of the coupling, the author derived appropriate reaction deletions using the new algorithm for target production in which the search space was divided into small cubes (CubeProd). Conclusions: The author developed a novel algorithm, CubeProd, to demonstrate that growth coupling is possible for most metabolites in S.cerevisiae under anaerobic conditions. This may imply that growth coupling is possible by reaction deletions for most target metabolites in any genome-scale constraint-based metabolic networks. The developed software, CubeProd, implemented in MATLAB, and the obtained reaction deletion strategies are freely available.
\end{abstract}

Keywords: metabolic network; flux balance analysis; growth coupling; linear programming; algorithm; constraint-based model 


\section{Background}

Metabolic network analysis such as flux balance analysis (FBA) is gaining importance in the metabolic engineering field. These models enable us to design and optimize metabolite producing microbes $[1,2,3]$. Coupling cellular growth with the production of a desired metabolite is a key constraint, known as growth coupling, in computational strain designs [4]. Unfortunately, growth coupling is not possible for every metabolite.

FBA is a widely used approach to solve mathematical models in metabolic engineering simulations. FBA assumes a pseudo-steady state in which the sum of incoming fluxes must equal the sum of outgoing fluxes for each internal metabolite [5]. Upper and lower bounds are initially given for each flux in original networks. However, more strict bounds may exist for each flux due to the influence of other fluxes. Such bounds for each flux can be determined by solving linear programming (LP) by maximizing or minimizing the flux. If a solution exists, one or more steady-state flux vector is obtained by solving this LP. Cell growth rate (GR), target metabolite production rate (PR), correspond to fluxes representing cell growth and target metabolite production, respectively. In conventional FBA simulation, GR is maximized to predict cell growth for reaction deletion strategies because it assumes that cells grow as fast as possible.

One of the simplest definitions of growth coupling is as follows. Under the condition that GR is maximized, growth coupling is achieved when PR exceeds a certain threshold, for example, $10 \%$ of the theoretical maximum production rate (TMPR). TMPR can be obtained by maximizing PR in the original LP. GR is not involved in the calculation of TMPR. In this study, to compare the performance with existing methods, growth yield $(\mathbf{G Y})$ and production yield $(\mathbf{P Y})$ ) are also utilized for another definition of growth coupling instead of GR and PR to consider the ratio of PR and substrate uptake rate (SUR), where $\mathrm{PY}=\mathrm{PR} / \mathrm{SUR}$ and $\mathrm{GY}=\mathrm{GR} / \mathrm{SUR}$ hold. In many cases, PY (or GY) of a flux vector with the maximum PR (resp. GR) is also the maximum.

Because LP may have multiple solutions, it is necessary to consider a range $[a, b]$ of PR when GR is maximized, where $a$ (or $b$ ) is the minimum (resp. maximum) PR under the condition that GR is maximized. It is to be noted that this is a bi-level optimization problem where GR is firstly maximized, and then PR is maximized or minimized. In this study, to ensure growth coupling even in the pessimistic cases, $a$ was evaluated for each reaction deletion strategy via flux variability analysis (FVA) [6]. FVA can determine the maximum and/or minimum values for the secondly optimized target under the condition that the firstly optimized target is maximize or minimized in bi-level optimization problems.

Many efficient solvers are available for LP, as LP is polynomial-time solvable. In computer science, a problem is considered to be efficiently solvable by an algorithm if the theoretical computational time is proportional to a polynomial function of the problem size. On the other hand, a problem is considered to be difficult to solve if the theoretical computational time of any algorithm is proportional to an exponential function of the problem size. NP-complete problems [7] are strongly believed that polynomial-time algorithms do not exist, and only exponential-time algorithms can solve it. It is impossible to solve large-scale NP-complete problems in many cases. 
Because the maximization problem for GR can be solved by LP, FBA simulations are efficiently possible even for genome-scale metabolic models. The fluxes calculated by FBA correspond to experimentally obtained fluxes [8]. Although ordinary differential equation-based models have more explanatory power, it is not suitable for genome-scale models because more detailed parameters are required. 0/1-values calculated by Boolean models do not exactly correspond to experimentally obtained fluxes but have complementary explanatory power to FBA-based simulation [9].

In metabolic engineering simulation using FBA, numerous computational methods have been developed to identify optimal design strategies including reaction deletions. OptKnock is a well-established method that identifies optimal design strategies [10]. OptKnock derives its design strategies through bi-level linear optimization using mixed integer LP. However, because mixed integer LP is an NP-complete problem [11], OptKnock cannot identify design strategies for many genome-scale networks. Many methods have been developed to overcome this problem (See Table 1). Each method has its own advantages, but they are not a panacea.

Elementary flux modes are particular steady-state flux vectors with a nondecomposability property. If two elementary flux modes differ only by a scalar factor, there are considered equivalent [12]. Elementary flux vectors (EFVs) are a generalization of elementary flux modes from cones to polyhedron in order to consider both lower and upper bounds of fluxes simultaneously [13, 14].

Because the assumption that cells grow as fast as possible is not always true in real cells, the EFV-based method evaluates PRs (or PYs) under various GRs (resp. GYs) in the growth coupling analysis. For this purpose, EFV-based methods have advantages as follows. When an EFV is given, GY and PY are uniquely determined by deleting reactions that are assigned 0 in the EFV because GR and PR are always proportional to SUR in this method. Because substrate uptake automatically enforces both cell growth and target metabolite production, GY and PY are strongly coupled in this method.

From a theoretical point of view, growth coupling is distinguished into strong coupling and weak coupling defined by Klamt and Mahadevan [12, 4]. Weak coupling means that a sufficiently large PY is achieved if GY is maximized or almost maximized. Strong coupling demands more, it additionally requires that PY must be high even if GY is low.

In 2017, von Kamp and Klamt developed an EFV-based method and showed that growth coupling is possible for more than $90 \%$ of all target metabolites by reaction deletions in Escherichia coli and Saccharomyces cerevisiae under aerobic conditions. They also provided similar results for Aspergillu niger, Corynebacterium glutamicum, and Synechocystis. They also showed that growth coupling is possible for more than $75 \%$ of all target metabolites in E. coli under anaerobic conditions. However, for S. cerevisiae under anaerobic conditions, the EFV-based method could only derive reaction deletion strategies for $3.9 \%$ of all metabolites. The low success ratio was because substrate glucose was almost completely converted into ethanol in the networks derived from EFVs for $S$. cerevisiae under anaerobic conditions [4]. They also showed that strong coupling is impossible for any gene-associated reaction deletion strategy that achieves a target production exceeding $10 \%$ of the theoretical maximum product yield (TMPY) for $94.6 \%$ of all metabolites, where TMPY is the maximum PY. 
This does not mean that growth coupling, which includes weak coupling, is impossible for $94.6 \%$ of all target metabolites. However, strong coupling by geneassociated reaction deletions is impossible. Therefore, growth coupling that is not strong coupling but weak coupling may be possible particularly if any reaction deletion is allowed. Due to the development of genetic engineering, deletion of any reaction may become possible in the near future. In this study, the author developed a novel algorithm, CubeProd, for finding reaction deletion strategies to demonstrate that growth coupling is possible for most metabolites in S.cerevisiae under anaerobic conditions. The proposed method also showed good results in Escherichia coli and/or aerobic conditions.

\section{Results}

\subsection{Model and environment}

The genome-scale models (GEM) that were used for growth coupling simulation for S. cerevisiae and Escherichia coli were iMM904 [15] and iJO1366 [16], respectively. If the target metabolite does not have an exchange reaction to the external environment, an auxiliary exchange reaction was temporarily added to the model to simulate the target metabolite production as in existing studies [4]. PR corresponds to the outgoing flux of this additional reaction or the exchange reaction. Because some exchange reactions including these additional reactions never excrete, $\mathrm{PR}$ of some target metabolites is zero, even if it is maximized. Such metabolites are excluded from the target metabolites. For example, the TMPR and TMPY are zero for 539 of the 1226 target metabolites in iMM904 under anaerobic conditions. Therefore, in the experiments, methods were applied only for the 687 remaining target metabolites. TPMY values used in the computational experiments refer to the data provided in [4]. Other information is summarized in Table 2(A).

For anaerobic simulation for S. cerevisiae and Escherichia coli cytochrome c oxidase and oxygen exchange reactions were removed in iMM904 and iJO1366, respectively. All the model configurations and the target metabolites were the same as in [4]. However, any reaction deletion strategy was allowed except for the EFV-based method.

All procedures in the computational experiments were implemented on a CentOS 7 machine with an Intel Xeon Processor with $2.30 \mathrm{GHz} 18 \mathrm{C} / 36 \mathrm{~T}$, and $128 \mathrm{~GB}$ memory. This workstation had CPLEX, COBRA Toolbox [17], and MATLAB.

\subsection{Performance of existing methods}

Theoretically, OptKnock can always find a desired reaction deletion strategy for any GEM if an unlimited amount of memory and time are available. However, it cannot complete the calculation in a realistic amount of time in many cases for a GEM. In the computational experiments, either OptKnock, GDLS, Redirector or GDBB could not find reaction deletion strategies for any of the 687 target metabolites in iMM904 under anaerobic conditions within 30 minutes. EMlio and FastPros seemed not available online as of July 2020. Because GridProd combined the ideas of pFBA and IdealKnock, the performance of CubeProd was compared with the EFV-based method and GridProd.

The difficulty with computing reaction deletion strategies for GEM is mainly due to the combinatorial explosion. When the number of reactions is $n$, the number of 
reaction deletion strategy candidates is $2^{n}$ if any reaction can be deleted. If there is an algorithm whose theoretical computation time is proportional to $n^{c}$ where $c$ is a small positive integer, a reaction deletion strategy enabling growth coupling can be computed for most cases even for GEM if such a reaction deletion strategy exists. However, identifying an optimal subnetwork that achieves the maximum PR when GR is maximized is an NP-complete problem; thus, it is often impossible to determine this information for GEM in a realistic time.

Because the EFV-based method reduces the search space by considering only strong coupling, it successfully determined the reaction deletion strategies for most target metabolites under aerobic conditions. However, it was not successful for $S$. cerevisiae under anaerobic conditions.

Computations were conducted for three different levels of demanded minimum product yield, $10 \%, 30 \%$, and $50 \%$ of the TMPY for each target metabolite [4]. The results are summarized in Table 2(B). The EFV-based method was very efficient for aerobic conditions, and efficient for iJO1366 under anaerobic conditions. However, it is necessary to develop a method that is efficient for iMM904 under anaerobic conditions.

The author previously developed a polynomial time algorithm to overcome the problem of combinatorial explosion, GridProd, which does not guarantee the identification of the optimal subnetwork, but can efficiently reveal appropriate deletion strategies for many target metabolites for E.coli under microaerobic conditions [18].

GridProd was applied to iMM904 under anaerobic conditions, and the results are summarized in Table 2(B). The performance of GridProd was moderate for iMM904 under anaerobic conditions and deserved to be improved to obtain a higher success ratio.

\subsection{Idea of CubeProd}

The idea of how GridProd finds reaction deletion strategies is as follows. In the growth coupling simulation using FBA, there are two special fluxes, GR and PR. Suppose that ideal point $=(\mathrm{GR}, \mathrm{PR})$ is values of $\mathrm{GR}$ and $\mathrm{PR}$ when the desired reaction deletions are given and GR is maximized. If the ideal point is initially known, it may be possible to obtain the fluxes that are the same as when the desired reaction deletions are given. If the number of ideal point candidates is proportional to a polynomial function of the problem size, this strategy can be conducted in a realistic computation time while it is not ensured that the desired reaction deletions are found. To this end, the notion of the ideal point was extended to the ideal ranges in GridProd.

GridProd considers solution space that is represented by a square whose horizontal and vertical axes correspond to $0 \leq \mathrm{GR} \leq \mathrm{TMGR}$ and $0 \leq \mathrm{PR} \leq \mathrm{TMPR}$, respectively, as shown in Fig. 1. The whole square corresponds to the whole solution space. Instead of maximizing GR or PR, GridProd adds two new constraints represented as small grids (squares) to the LP whose horizontal and vertical axes correspond to small ranges of GR and PR, respectively. GridProd divides the solution space into small grids and solves LPs twice for each grid.

The first LP is obtained by adding the two new constraints to the original LP, and GridProd obtains a flux vector by solving the first LP. Reactions that are not used 
in this flux vector are treated as the knockout candidates and deleted in the second LP. The second LP is obtained by assigning 0 to the reactions determined in the first LP to the original LP, where the two new constraints are not included. Then, the knockout strategy of the grid whose PR is the best is adopted as the output of GridProd. Finally, FVA was conducted for the obtained reaction deletion strategy to evaluate PR in the most pessimistic case. GridProd gradually moves the grid to scan the solution space. When the horizontal and vertical edges of the whole square are divided into $P$ small edges, respectively, the number of small squares (grids) is $P^{2}$.

Because LP is solvable in polynomial time of the numbers of reactions and metabolites, the theoretical computation time of GridProd is polynomial of the numbers of reaction and metabolites times $P^{2}$. Therefore, GridProd is fast enough to be applied to genome-scale networks.

Although the knockouts of these reactions obtained in the first LP do not always realize the ranges of GR and PR of attention in the solution of the second LP in each grid as shown in Fig. 1(B), experimental results showed that it works well if the sizes of the grids are small enough. However, if the sizes of grids are too small, in other words, $P$ is too large, the computational cost becomes too expensive, although $P^{2}$ is polynomial of $P$. In the first LP of GridProd, the sum of absolute values of fluxes is minimized because the flux vectors with more 0s obtained in the first LP are more reproducible in the second LP. In the second LP of GridProd, GR is maximized to verify whether growth coupling is achieved.

However, because multiple valid flux vectors may exist in the grid that corresponds to the ideal range, it is not guaranteed that the GridProd always obtains the optimal flux vector. Theoretically, if the grid corresponding to the ideal range is small enough and the flux vector with the GR and PR ranges is uniquely determined in the first LP, then the flux vectors obtained by the first and second LPs are the same as those obtained by the optimal reaction deletions. However, if the grids are too small, the computation time becomes too large. Therefore, in GridProd, each grid is relatively small but not too small so that GridProd can achieve relatively high PR and short computational time.

However, GridProd was not efficient enough for S.cerevisiae under anaerobic conditions as shown in Table 2(B). When GridProd could not derive appropriate reaction deletion strategies, the flux vector obtained by the first LP were often quite different from those obtained by the second LP. One of the promising methods for overcoming this problem is to decrease the number of optimal solutions by adding other constraints for each LP.

To this end, while GridProd imposes the following two constraints

$$
\begin{aligned}
& T M G R \times p \times(i-1) \leq G R \leq T M G R \times p \times i \\
& T M P R \times p \times(j-1) \leq P R \leq T M P R \times p \times j,
\end{aligned}
$$

CubeProd imposes the following three constraints

$$
\begin{aligned}
& T M G R \times p \times(i-1) \leq G R \leq T M G R \times p \times i \\
& T M P R \times p \times(j-1) \quad \leq P R \leq T M P R \times p \times j, \\
& T M T F \times p \times(k-1) \leq T F \leq T M T F \times p \times k,
\end{aligned}
$$


where TMTF represents the theoretical maximum total flux, that is the maximum value of the sum of the absolute values of fluxes, for all integers $1 \leq i, j, k \leq P$, where $P=1 / p$ holds. In addition to the above modification, after solving the first LP, CubeProd conducts FVA instead of the second LP for each grid. This makes CubeProd fails to find the desired reaction deletions less often than GridProd. The above two points are the main differences from GridProd. Furthermore, for CubeProd with $\mathrm{P}$, the computational experiments were conducted for all $c$ with $5 \leq c \leq \mathrm{P}$, while GridProd was conducted only for $\mathrm{P}$. The influence of this difference for $\mathrm{P}$ can be seen in Fig. 2. The LP of CubeProd minimizes the sum of the absolute values of fluxes as similar to GridProd. $P$ is called the resolution in GridProd and CubeProd.

\subsection{Performance of CubeProd}

The success ratios of CubeProd for 687 target metabolites of IMM904 under anaerobic conditions were $91.3 \%, 69.6 \%$, and $47.6 \%$ when the demanded minimum yields were $10 \%, 30 \%$, and $50 \%$ of the TMPY, respectively when $P \leq 15$. The results for aerobic conditions and Escherichia coli are also summarized in Table 2(B).

Fig. 2 shows the percentages of producible organic metabolites by the reaction deletion strategies obtained by CubeProd that achieved growth coupling for the 687 target metabolites of iMM904 for different resolutions (cube sizes) and demanded minimum yield levels. The horizontal axis corresponds to different resolutions of CubeProd. The blue, red, black, and green lines correspond to the demanded minimum yield levels of $1 \%, 10 \%, 30 \%$, and $50 \%$, respectively. Each line is shown with a monotone increase because the experiments were conducted for every $c$ with $5 \leq c \leq P$ for given $\mathrm{P}$. The average computation time for each producible metabolite was $39.7 \mathrm{~s}$ and $943.7 \mathrm{~s}$ for $P=5$ and $P=15$, respectively.

To verify the performance of CubeProd for more recent GEM, CubeProd was applied to yeast7.8 [19], which was published in 2019. For anaerobic conditions, ferrocytochrome-c: oxygen oxidoreductase was removed. There were 1758 target metabolites whose TMPRs were not zero. The success ratios of CubeProd under anaerobic conditions were $89.9 \%, 74.5 \%$, and $45.9 \%$ when the demanded minimum PRs were $10 \%, 30 \%$, and $50 \%$ of the TMPR, respectively when $P \leq 15$. For aerobic conditions, there were 1760 target metabolites, whose TMPRs were not zero. The success ratios were $76.2 \%, 67.3 \%$, and $57.8 \%$, respectively.

Computational experiments were also conducted for iMM904 under anaerobic conditions for the cases where spontaneous reactions were not allowed to be deleted. In iMM904, three reactions AOBUTDs, G5SADs, PHCHGSm, are notated as spontaneous. When these reactions are excluded from deletion candidates, the success ratios of CubeProd for 687 target metabolites were $77.0 \%, 60.1 \%$, and $42.4 \%$ when the demanded minimum PYs were $10 \%, 30 \%$, and $50 \%$ of the TMPY, respectively when $P \leq 15$.

In each LP in the above experiments, it was necessary to consider that G5SADs and PHCHGSm are reversible, while AOBUTDs is irreversible. The author considered the following four cases (1) both G5SADs and PHCHGSm have positive fluxes, (2) both G5SADs and PHCHGSm have negative fluxes, (3) G5SADs and PHCHGSm have positive and negative fluxes, respectively, (4) G5SADs and 
PHCHGSm have negative and positive fluxes, respectively. They were represented as additional constraints in each LP to find reaction deletion candidates that exclude spontaneous reactions.

\subsection{Comparing CubeProd with GridProd}

CubeProd is an extension of GridProd. To explain why CubeProd worked better than GridProd in the computational experiments, the concept of CubeProd was compared to GridProd using the following examples on the toy model of the metabolic network shown in Fig. $3(\mathrm{~A}) .\{\mathrm{R} 1, \ldots, \mathrm{R} 8\}$ and $\{\mathrm{C} 1, \ldots, \mathrm{C} 5\}$ are sets of reactions and metabolites, respectively. $\mathrm{R} 1$ is a source exchange reaction such as glucose or oxygen uptake. R3 is the biomass objective function consuming $\mathrm{C} 2$ that corresponds to GR. R8 is the exchange reaction of the target metabolite C5. All of the reactions are irreversible. $[a, b]$ indicates that $a$ and $b$ are the lower and upper bounds of the flux for the corresponding reaction, respectively. R1, R3, and R8 are not allowed to be deleted.

Under the assumption that GR is maximized, the optimal design strategy for the worst-case-scenario by FVA is to "delete R2" or "delete R2 and R7." as shown in Fig. 3 (B). When some of $\{\mathrm{R} 4, \mathrm{R} 5, \mathrm{R} 6\}$ is deleted in addition to $\mathrm{R} 2, \mathrm{GR}=0$ is obtained and the required minimum growth is not satisfied.

In the following, the author investigates whether CubeProd and GridProd can identify the optimal strategies in this example. For simplicity of explanation, the TAF of only internal reactions is considered and $\epsilon$ is zero. Fig.3(C) shows that GridProd cannot predict appropriate reaction deletions when $4 \leq \mathrm{GR} \leq 6$ and $4 \leq$ $\mathrm{PR} \leq 6$ are considered. Even when the resolution is infinity and $\mathrm{GR}=5$ and $\mathrm{PR}=5$ are considered, GridProd cannot find the optimal strategy.

CubeProd predicts the design strategy for each small ranges of GR, PR, and the total amount of fluxes (TAF). For example, suppose that $5-\epsilon \leq \mathrm{GR} \leq 5+\epsilon, 5-\epsilon \leq$ $\mathrm{PR} \leq 5+\epsilon$, and $15-\epsilon \leq \mathrm{TAF} \leq 15+\epsilon$ are considered, where $\epsilon$ is a small positive constant. Then, the flux must satisfy $\mathrm{R} 2+\mathrm{R} 5=5, \mathrm{R} 5+\mathrm{R} 7=5, \mathrm{R} 2+3 \cdot \mathrm{R} 5+\mathrm{R} 7$ $=15$, and $(\mathrm{R} 2, \mathrm{R} 4, \mathrm{R} 5, \mathrm{R} 6, \mathrm{R} 7)=(0,5,5,5,0)$ is uniquely determined. Therefore, when $\mathrm{R} 2$ and $\mathrm{R} 7$ are deleted, and $(\mathrm{GR}, \mathrm{PR})=(5,5)$ is obtained as shown in Fig.3 (D) under the condition that GR is maximized.

However, suppose that the resolution of CubeProd is not large enough. For example, suppose that $4 \leq \mathrm{GR} \leq 6,4 \leq \mathrm{PR} \leq 6,12 \leq \mathrm{TAF} \leq 18$ are considered. In this case, $(\mathrm{R} 2, \mathrm{R} 4, \mathrm{R} 5, \mathrm{R} 6, \mathrm{R} 7)=(6,0,0,0,6)$ also satisfies the constraints in addition to $(\mathrm{R} 2, \mathrm{R} 4, \mathrm{R} 5, \mathrm{R} 6, \mathrm{R} 7)=(0,4,4,4,0)$. If CubeProd selects the former, $(\mathrm{GR}, \mathrm{PR})=(10,0)$ is obtained, although $(\mathrm{GR}, \mathrm{PR})=(5,5)$ is obtained if CubeProd selects the latter.

Thus, for the network of Fig. 3 (A), GridProd cannot find the appropriate strategies, but CubeProd can always find it if the resolution is large enough.

\subsection{Size of deletion}

While the numbers of the reaction deletions derived through the EFV-based method were less than $3 \%$ of all reactions, those derived through CubeProd were $78.9 \%$ on average for iMM904 under anaerobic conditions.

Fig. 4 represents the number of times each reaction was deleted when CubeProd succeeded in finding deletion strategies for iMM904 under anaerobic conditions. 
548 of the 1577 iMM904 reactions were deleted for all 627 cases where CubeProd found appropriate deletion strategies. 173 of the 1577 iMM904 reactions were never deleted. On average, 1245 of the 1577 reactions (78.9\%) were deleted for each target metabolite.

\section{Discussion}

The basic concept of the proposed method is to balance the computation time and the success ratio of production. If infinite computation time is allowed, then the deletion strategy to achieve growth coupling can always be obtained using optknock, as long as there is a solution. However, since the problem of finding reaction deletions that maximize PR under the condition that GR is maximized is NP-complete, it requires computational time proportional to the exponential function of the problem size. Therefore, as the network becomes larger, the computation does not complete in a realistic time.

To overcome the problem of increasing computation time, many methods, including optknock, limit the number of deletions. Even if the problem is NP-complete, if the number of reactions is $n$ and the number of deletions is limited to $c$, which is a small positive integer, it can be solved in a computation time proportional to $n^{c}$, which is polynomial of $n$ and solvable efficiently. This speed-up can be achieved by narrowing the solution space. The concept of strong coupling was proposed out of practical necessity in metabolic engineering, but it also serves the purpose of narrowing the solution space.

The EFV-based method calculates reaction deletion strategies that achieve strong coupling by finding EFVs that simultaneously contain growth and production. Due to the nature of EFV-based method, it is difficult to find reaction deletions that achieve a weak coupling but not a strong coupling. This may be the first reason why CubeProd worked well in yeast anaerobic conditions, and the EFV-based method did not. The second reason may be that the EFV-based method limits deletions to gene-associated reactions, whereas the CubeProd targets all reactions (or all non-spontaneous reactions). Because of the large number of non-gene associated reactions, it is difficult to limit deletions to non-gene associated reactions due to the nature of Cubeprod. On the other hand, it is promising to extend Cubeprod to compute reaction deletions that achieve strong coupling.

Because the strategies by CubeProd include non-gene associated reactions and the average deletion size of CubeProd is $78.9 \%$, readers may think that the strategies by CubeProd are not realistic.

It is not practical to remove many reactions with traditional genetic engineering techniques, but if it is based on DNA synthesis, the fewer reactions that are left, the better. Fortunately, DNA synthesis technologies are consistently being improved [20]. Designing short synthetic DNA may replace the method of knocking out genes from the original long genome to produce metabolites. In such a case, the number of genes included in the design of synthetic DNA should be as small as possible because of the experimental effort and time required.

The success ratio in the computer experiments that prohibited the removal of spontaneous reactions was $77.0 \%$ when the demanded minimum PYs were $10 \%$ of the TMPYs for S.cerevisiae under anaerobic conditions. Although this was lower 
than the $91.3 \%$ success ratio compared to allowing all reactions to be deleted, it was significant to be able to demonstrate a deletion strategy for many target compounds.

In GEMs, each gene associated reaction is assigned a Boolean function that takes genes as inputs. In traditional metabolic engineering, reaction deletion strategies are achieved through gene deletions via these Boolean functions. However, whether each reaction is gene-associated is according to current knowledge and technologies, which may be updated in the future. If the reaction is not spontaneous, molecular biology development may lead to the development of the control methods in the future, even if it is never gene-associated.

Therefore, it is important to show the possibility of growth coupling when any reaction deletion is allowed. Computational experiments using CubeProd showed that growth coupling is possible for most metabolites, even in S. cerevisiae under anaerobic conditions. On the other hand, the performance of CubeProd was moderate for Escherichia coli under anaerobic conditions. However, there is room for improvement by increasing $\mathrm{P}$ and introducing another variable as an axis. Extending the algorithm framework is also promising by utilizing a fact that if a solution is not found in the first LP in a range, then no solution can be found by dividing the range further. This will speed up the process, allow CubeProd to examine larger P's, and increase the success ratio. These are left for future work.

\section{Conclusion}

The author developed a novel algorithm, CubeProd, and demonstrated that growth coupling is possible for most metabolites in S.cerevisiae under anaerobic conditions, for which the existing methods were not efficient. For example, either GridProd or the EFV-based method could not determine the reaction deletions for producing L-alanine for S.cerevisiae under anaerobic conditions, but CubeProd could.

The determined reaction deletion strategies by CubeProd include non-geneassociated reactions and gene-associated reactions. Current knowledge determines whether each reaction is gene-associated, and this knowledge may be updated or refined in the future. Thus, it is also important to consider reaction deletion strategies including non-gene-associated reactions.

CubeProd determines reaction deletion strategies that achieve growth coupling for genome-scale metabolic networks by extending the concept of GridProd [18], which was developed by the author. GridProd divides the solution space of optKnock into $P^{2}$ small grids and conducts LPs twice for each grid, where $P$ is a positive integer parameter. The first LP identifies the reactions to be deleted. The second LP calculates the PR of the target metabolite when the determined reactions by the first LP are deleted and GR is maximized for each grid. The deletion strategy of the grid whose PR is highest is then adopted as the GridProd solution. Finally, the minimum PR was evaluated under the condition that GR is maximized for the reaction deletions using FVA. The area size of each grid in GridProd is $(p \times$ $T M G R) \times(p \times T M P R)$, where TMGR and TMPR are the theoretical maximum growth rate and theoretical maximum production rate, respectively.

In addition to $(p \times T M G R) \times(p \times T M P R)$, CubeProd divides the solution space by $(p \times T M G R) \times(p \times T M P R) \times(p \times T M T F)$, where TMTF is the theoretical maximum total flux, which is the maximum value of the sum of absolute values of fluxes. 


\section{Methods}

The pseudo-code of CubeProd is as follows.

Procedure CubeProd(target, $P$ )

$T M G R=\max v_{\text {growth }}$

s.t. $\Sigma S_{i, j} \cdot v_{j}=0$

$L B_{j} \leq v_{j} \leq U B_{j}, v_{\text {glc_uptake }} \geq-G U R$

$\mathrm{v}_{\text {atp_main }} \geq A T P, v_{\text {cyt }}=0$

$T M P R=\max v_{\text {target }}$

s.t. $\Sigma S_{i, j} \cdot v_{j}=0$

$L B_{j} \leq v_{j} \leq U B_{j}, v_{\text {glc_uptake }} \geq-G U R$

$\mathrm{v}_{\text {atp_main }} \geq A T P, v_{\text {cyt }}=0$

$v_{\text {growth }} \geq v_{\text {growth }}^{\text {min }}$

$T M T F=\max \Sigma\left|v_{j}\right|$

s.t. $\Sigma S_{i, j} \cdot v_{j}=0$

$L B_{j} \leq v_{j} \leq U B_{j}, \mathrm{v}_{\text {glc_uptake }} \geq-G U R$

$\mathrm{v}_{a t p \_m a i n} \geq A T P, v_{c y t}=0$

$v_{\text {growth }} \geq v_{\text {growth }}^{\text {min }}$

for $i=1$ to $P$ do

$$
\begin{aligned}
& \text { biomass } L B=T M G R \times P \times(i-1) \\
& \text { biomassUB }=T M G R \times P \times i \\
& \text { for } j=1 \text { to } P \text { do } \\
& \text { target } L B=T M P R \times P \times(j-1) \\
& \text { target } U B=T M P R \times P \times j \\
& \text { for } k=1 \text { to } P \text { do } \\
& \text { totalFluxLB }=T M T F \times P \times(k-1) \\
& \text { totalFluxUB }=T M T F \times P \times k \\
& \% \text { The LP for }(i, j, k) \text {. } \\
& R_{\text {deleted }}(i, j, k) \text { is such that } \\
& \min \Sigma t_{j} \\
& \text { s.t. } \Sigma S_{i, j} \cdot v_{j}=0 \\
& L B_{j} \leq v_{j} \leq U B_{j},-t_{j} \leq v_{j} \leq t_{j} \\
& v_{\text {glc_uptake }} \geq-G U R, v_{\text {atp_main }} \geq A T P, v_{\text {cyt }}=0 \\
& \text { biomass } L B \leq v_{\text {growth }} \leq \text { biomass } U B \\
& \text { target } L B \leq v_{\text {target }} \leq \text { target } U B \\
& R_{\text {deleted }}=\left\{v_{j} \mid v_{j}<10^{-5}\right\}
\end{aligned}
$$

if the LP is not feasible

$$
R_{\text {deleted }}(i, j, k)=\phi
$$

$\%$ FVA with $R_{\text {deleted }}(i, j, k)$.

$v_{\text {target }}$ is the minimum value satisfying the following

$\max v_{\text {growth }}$

s.t. $\Sigma S_{i, j} \cdot v_{j}=0\left(v_{\text {target }} \in v\right)$

$L B_{j} \leq v_{j} \leq U B_{j}$ for $\left\{j \mid v_{j} \notin R_{\text {deleted }}(i, j, k)\right\}$

$v_{j}=0$ for $\left\{j \mid v_{j} \in R_{\text {deleted }}(i, j, k)\right\}$

$v_{\text {glc_uptake }} \geq-G U R, v_{\text {atp_main }} \geq A T P, v_{\text {cyt }}=0$

if $v_{\text {growth }} \geq v_{\text {growth }}^{\text {min }}$

$$
\operatorname{PR}(i, j, k)=v_{\text {target }}
$$




$$
\begin{gathered}
\text { else } \\
P R(i, j, k)=0 \\
(i, j, k)=\operatorname{argmax}(\operatorname{PR}(i, j, k)) \\
\text { return } R_{\text {deleted }}(i, j, k), \operatorname{PR}(i, j, k)
\end{gathered}
$$

In the above pseudo-code, the TMGR, TMPR, and TMTF are calculated first. $S_{i, j}$ is the stoichiometric matrix. $L B_{j}$ and $U B_{j}$ are the lower and upper bounds of $v_{j}$, respectively, which represent the flux of the $j$ th reaction.

$v_{\text {glc_uptake }}$, and $v_{\text {atp_main }}$ are the lower bounds of the uptake rate of glucose (GUR), and ATP maintenance demand, respectively. $v_{\text {growth }}^{\text {min }}$ is the minimum required cell growth rate.

In each cube, LP and FVA are conducted. "biomassLB" and "biomassUB" represent the lower and upper bounds of GR, respectively. "target $L B$ " and "targe$t U B$ " represent the lower and upper bounds of PR, respectively. "totalFluxLB" and "totalFluxUB" represent the lower and upper bounds of total amounts of fluxes, respectively. These values are used as the constraints in the LP. Each cube is represented by the three constraints, "biomassLB $\leq v_{\text {growth }} \leq$ biomassUB," "target $L B \leq v_{\text {target }} \leq \operatorname{target} U B$," and "totalFlux $L B \leq \Sigma\left|v_{j}\right| \leq$ totalFluxUB." $T M P R \times p, T M G R \times p$, and $T M T F \times p$, represent the length of the first, second and third dimension for each cube, respectively.

In the solution of the LP, a set of reactions whose fluxes are nearly 0 (less than $10^{-5}$ ) are represented as $R_{\text {deleted }}$, which is used as a set of deleted reactions in the FVA. In the FVA, the fluxes of the reactions included in $R_{\text {deleted }}$ were forced to be 0 . If the obtained PR is more than or equal to $v_{\text {growth }}^{\text {min }}$ in the solution of the FVA, the value of $\mathrm{PR}$ is stored to $P R(i, j, k)$. Otherwise 0 is stored. Finally, the $(i, j, k)$ yielding the maximum value in $P R(i, j, k)$ is searched, and the corresponding $R_{\text {deleted }}(i, j, k)$ and $P R(i, j, k)$ are obtained. The minimum PR from FVA for $R_{\text {deleted }}(i, j, k)$ are also calculated.

\section{List of abbreviation}

Flux Balance Analysis (FBA)

Flux Variability Analysis (FVA)

Linear Programming (LP)

Growth Rate (GR)

Growth Yield (GY)

Production Rate (PR)

Production Yield (PY)

Theoretical Maximum Growth Rate (TMGR)

Theoretical Maximum Growth Yield (TMGY)

Theoretical Maximum Production Rate (TMPR)

Theoretical Maximum Production Yield (TMPY)

Theoretical Maximum Total Flux (TMTF)

Glucose Uptake Rate (GUR)

Elementary Flux Vector (EFV) 
Declarations

Ethics approval and consent to participate

Not applicable

Consent to publish

Not applicable

Availability and data and materials

All source codes and data are included in the additional supporting file.

Competing interests

The author declares that he has no competing interests.

Funding

TT was partially supported by grants from JSPS, KAKENHI \#16K00391, \#16H02485, and \#20H04242. No

funding body played any roles in the design of the study and collection, analysis, and interpretation of data and in writing the manuscript

Author's contributions

This work has been done only by TT.

Acknowledgements

I appreciate the editor, reviewers, my colleagues. and my family.

Figures

Figure 1 Idea of GridProd. (A)Heat map representation of the acetate production by GridProd simulation with $\mathrm{P}=15$ for iMM904 under anaerobic conditions with required minimum growth

0.01 . The horizontal and vertical axes correspond to the constraints for GR and PR, respectively. (B)Theoretical maximum values, ranges of the best grid, and the results of the 1st and 2nd LPs for GR and PR, and the minimum PR by FVA.

Figure 2 Success ratio of CubeProd for each resolution and demanded minimum yield. The success ratio of CubeProd in identifying reaction deletion strategies that could achieve growth coupling for 687 target metabolites of iMM904 under anaerobic conditions at each resolution $(\leq P)$ and demanded minimum yield.

Figure 3 Comparing CubeProd with GridProd (A)A toy example in which CubeProd can identify the optimal strategy, but GridProd cannot. (B)The flux vectors for reaction deletions obtained by FVA minimizing R8 under the condition that R3 is maximized. (C)GridProd cannot find appropriate reaction deletions. (D)CubeProd can find appropriate reaction deletions if the resolution is large enough (cubes are small enough).

Figure 4 Deletion frequency of reactions The percentage of occurrences each reaction was deleted in the CubeProd strategy. 
Tables

Table 1 List of existing methods for calculating reaction deletion strategies with growth coupling.

\begin{tabular}{|ll|l|}
\hline Method & & Approach \\
\hline OptKnock & {$[10]$} & Mixed integer linear programming \\
GDLS & {$[21]$} & Local search with multiple search paths \\
EMILio & {$[22]$} & Iterative linear programs \\
Redirector & {$[23]$} & Iterative linear programs \\
GDBB & {$[24]$} & Genetic design through branch and bound \\
pFBA & {$[25]$} & Parsimonious metabolic network topology \\
IdealKnock & {$[26]$} & Ideal-type flux distribution, ideal point \\
FastPros & {$[27]$} & Iterative screening \\
GridProd & {$[18]$} & Integration of the ideas of IdealKnock and pFBA \\
EFV-based & {$[4]$} & Elementary flux vector-based method \\
\hline
\end{tabular}

Table 2 (A)Datasets used in the computational experiments. (B)Feasibility of growth coupling for Escherichia coli and S. cerevisiae under aerobic and anaerobic conditions demonstrated by the EFV-based method, GridProd, and CubeProd. The feasibility was demonstrated by calculating reaction deletion strategies. Percentage of producible organic metabolites is shown for each required minimum yield.

\begin{tabular}{|c|c|c|c|c|c|}
\hline & Conditions & \#reactions & \#metabolites & $\begin{array}{c}\text { \#target } \\
\text { metabolites }\end{array}$ & $\begin{array}{c}\text { Minimum } \\
\text { growth }\end{array}$ \\
\hline iJO1366 & $\begin{array}{c}\text { aerobic } \\
\text { anaerobic }\end{array}$ & 2583 & 1805 & $\begin{array}{l}954 \\
935\end{array}$ & 0.01 \\
\hline iMM904 & $\begin{array}{c}\text { aerobic } \\
\text { anaerobic }\end{array}$ & 1577 & 1226 & $\begin{array}{l}687 \\
687\end{array}$ & 0.01 \\
\hline
\end{tabular}

(A)

\begin{tabular}{|c|c|c|c|c|c|}
\hline & & & EFV-based & GridProd $(P=15)$ & CubeProd $(\mathrm{P} \leq 15)$ \\
\hline \multirow{6}{*}{ iJO1366 } & \multirow{3}{*}{ aerobic } & $10 \%$ & 99.4 & 39.0 & 96.8 \\
\hline & & $30 \%$ & 99.0 & 27.5 & 93.5 \\
\hline & & $50 \%$ & 96.5 & 21.3 & 54.8 \\
\hline & \multirow{3}{*}{ anaerobic } & $10 \%$ & 77.4 & 10.1 & 52.3 \\
\hline & & $30 \%$ & 55.6 & 5.8 & 30.2 \\
\hline & & $50 \%$ & 30.9 & 4.5 & 19.4 \\
\hline \multirow{6}{*}{ iMM904 } & \multirow{3}{*}{ aerobic } & $10 \%$ & 99.3 & 35.2 & 92.0 \\
\hline & & $30 \%$ & 98.3 & 14.0 & 37.3 \\
\hline & & $50 \%$ & 97.4 & 6.0 & 11.1 \\
\hline & \multirow{3}{*}{ anaerobic } & $10 \%$ & 3.9 & 51.7 & 91.3 \\
\hline & & $30 \%$ & 1.7 & 34.2 & 69.6 \\
\hline & & $50 \%$ & 1.3 & 19.8 & 47.6 \\
\hline
\end{tabular}

(B)

\section{Additional Files}

sourceCode.zip

"CubeProd.m" is the MATLAB source code of CubeProd. "example.m" is an example code to show how to use CubeProd. The detailed description is available in the comments of each file. The folder "deletionStrategies" contains the reaction deletion strategies obtained by CubeProd for iMM904, iJO1366, and yeast7.8 under anaerobic and aerobic conditions. Each subfolder contains data of the achieved minimum production yield, reactions to be deleted, target metabolites whose TMPY were not zero, and TMPY (TMPR for yeast7.8).

iMM904anaerobic.zip

The folder contains the data of reaction deletion strategies, the achieved minimum production yield, the target metabolites whose TMPY were not zero, and TMPY for iMM904 under anaerobic conditions.

iMM904aerobic.zip

The folder contains the data of reaction deletion strategies, the achieved minimum production yield, the target metabolites whose TMPY were not zero, and TMPY for iMM904 under aerobic conditions.

ijO1366anaerobic.zip

The folder contains the data of reaction deletion strategies, the achieved minimum production yield, the target metabolites whose TMPY were not zero, and TMPY for iJO1366 under anaerobic conditions. 
iJO1366aerobic.zip

The folder contains the data of reaction deletion strategies, the achieved minimum production yield, the target metabolites whose TMPY were not zero, and TMPY (TMPR for for iJO1366 under aerobic conditions.

yeast780anaerobic.zip

The folder contains the data of reaction deletion strategies, the achieved minimum production yield, the target metabolites whose TMPY were not zero, and TMPR for yeast7.8 under anaerobic conditions.

yeast780aerobic.zip

The folder contains the data of reaction deletion strategies, the achieved minimum production yield, the target metabolites whose TMPY were not zero, and TMPR for yeast7.8 under aerobic conditions.

References

1. Keasling, J.D.: Manufacturing molecules through metabolic engineering. Science 330(6009), 1355-1358 (2010)

2. Becker, J., Wittmann, C.: Advanced biotechnology: Metabolically engineered cells for the bio-based production of chemicals and fuels, materials, and health-care products. Angewandte Chemie International Edition 54(11), 3328-3350 (2015)

3. Lee, S.Y., Kim, H.U.: Systems strategies for developing industrial microbial strains. Nature biotechnology 33(10), 1061 (2015)

4. von Kamp, A., Klamt, S.: Growth-coupled overproduction is feasible for almost all metabolites in five major production organisms. Nature communications 8, 15956 (2017)

5. Orth, J.D., Thiele, I., Palsson, B.Ø.: What is flux balance analysis? Nature biotechnology 28(3), 245-248 (2010)

6. Maranas, C.D., Zomorrodi, A.R.: Optimization Methods in Metabolic Networks. John Wiley \& Sons, ??? (2016)

7. Arora, S., Barak, B.: Computational Complexity: a Modern Approach. Cambridge University Press, ??? (2009)

8. Varma, A., Palsson, B.O.: Stoichiometric flux balance models quantitatively predict growth and metabolic by-product secretion in wild-type escherichia coli w3110. Applied and environmental microbiology 60(10), 3724-3731 (1994)

9. Tamura, T., Lu, W., Song, J., Akutsu, T.: Computing minimum reaction modifications in a boolean metabolic network. IEEE/ACM transactions on computational biology and bioinformatics 15(6), 1853-1862 (2017)

10. Burgard, A.P., Pharkya, P., Maranas, C.D.: Optknock: a bilevel programming framework for identifying gene knockout strategies for microbial strain optimization. Biotechnology and bioengineering 84(6), 647-657 (2003)

11. Schrijver, A.: Theory of Linear and Integer Programming. John Wiley \& Sons, ??? (1998)

12. Klamt, S., Mahadevan, R.: On the feasibility of growth-coupled product synthesis in microbial strains. Metabolic engineering 30, 166-178 (2015)

13. Klamt, S., Regensburger, G., Gerstl, M.P., Jungreuthmayer, C., Schuster, S., Mahadevan, R., Zanghellini, J. Müller, S.: From elementary flux modes to elementary flux vectors: Metabolic pathway analysis with arbitrary linear flux constraints. PLoS computational biology 13(4) (2017)

14. Urbanczik, R.: Enumerating constrained elementary flux vectors of metabolic networks. IET systems biology 1(5), 274-279 (2007)

15. Mo, M.L., Palsson, B.Ø., Herrgård, M.J.: Connecting extracellular metabolomic measurements to intracellular flux states in yeast. BMC systems biology 3(1), 37 (2009)

16. Orth, J.D., Conrad, T.M., Na, J., Lerman, J.A., Nam, H., Feist, A.M., Palsson, B. $\varnothing$. : A comprehensive genome-scale reconstruction of escherichia coli metabolism-2011. Molecular systems biology 7(1), 535 (2011)

17. Heirendt, L., Arreckx, S., Pfau, T., Mendoza, S.N., Richelle, A., Heinken, A., Haraldsdóttir, H.S., Wachowiak, J., Keating, S.M., Vlasov, V., et al.: Creation and analysis of biochemical constraint-based models using the cobra toolbox v. 3.0. Nature protocols 14(3), 639-702 (2019)

18. Tamura, T.: Grid-based computational methods for the design of constraint-based parsimonious chemical reaction networks to simulate metabolite production: Gridprod. BMC bioinformatics 19(1), 325 (2018)

19. Sánchez, B.J., Li, F., Kerkhoven, E.J., Nielsen, J.: Slimer: probing flexibility of lipid metabolism in yeast with an improved constraint-based modeling framework. BMC systems biology 13(1), 4 (2019)

20. Kosuri, S., Church, G.M.: Large-scale de novo dna synthesis: technologies and applications. Nature methods 11(5), 499-507 (2014)

21. Lun, D.S., Rockwell, G., Guido, N.J., Baym, M., Kelner, J.A., Berger, B., Galagan, J.E., Church, G.M.: Large-scale identification of genetic design strategies using local search. molecular systems biology 5(1), 296 (2009)

22. Rockwell, G., Guido, N.J., Church, G.M.: Redirector: designing cell factories by reconstructing the metabolic objective. PLoS Comput Biol 9(1), 1002882 (2013)

23. Yang, L., Cluett, W.R., Mahadevan, R.: Emilio: a fast algorithm for genome-scale strain design. Metabolic engineering 13(3), 272-281 (2011)

24. Egen, D., Lun, D.S.: Truncated branch and bound achieves efficient constraint-based genetic design. Bioinformatics 28(12), 1619-1623 (2012)

25. Lewis, N.E., Hixson, K.K., Conrad, T.M., Lerman, J.A., Charusanti, P., Polpitiya, A.D., Adkins, J.N., Schramm, G., Purvine, S.O., Lopez-Ferrer, D., et al.: Omic data from evolved e. coli are consistent with computed optimal growth from genome-scale models. Molecular systems biology 6(1), 390 (2010)

26. Gu, D., Zhang, C., Zhou, S., Wei, L., Hua, Q.: Idealknock: a framework for efficiently identifying knockout strategies leading to targeted overproduction. Computational biology and chemistry 61, 229-237 (2016)

27. Ohno, S., Shimizu, H., Furusawa, C.: Fastpros: screening of reaction knockout strategies for metabolic engineering. Bioinformatics 30(7), 981-987 (2014) 


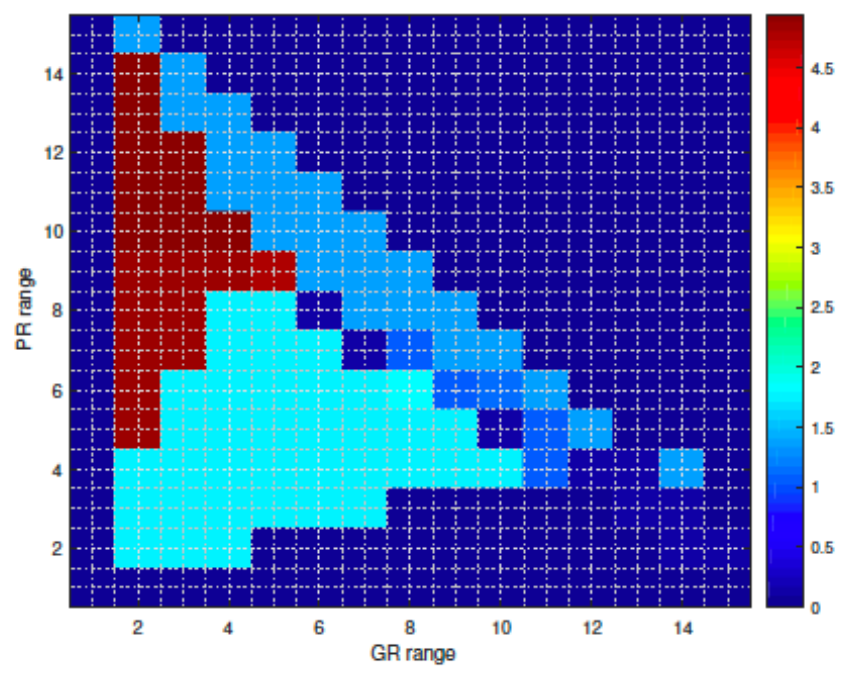

(A)

\begin{tabular}{|c|c|c|c|c|c|}
\hline & Theoretical maximum & Constraints of the best grid & 1st LP & 2nd LP & FVA \\
\hline GR & 0.208 & $0.0138 \leq \mathrm{GR} \leq 0.0277$ & 0.0138 & 0.050 & \\
PR & 9.07 & $6.65 \leq \mathrm{PR} \leq 7.26$ & 6.65 & 4.94 & 4.94 \\
\hline
\end{tabular}

(B)

Figure 1

(A)Heat map representation of the acetate production by GridProd simulation with $\mathrm{P}=15$ for iMM904 under anaerobic conditions with required minimum growth 0.01 . The horizontal and vertical axes correspond to the constraints for GR and PR, respectively. (B)Theoretical maximum values, ranges of the best grid, and the results of the 1st and 2nd LPs for GR and PR, and the minimum PR by FVA. 


\section{Success ratio of CubeProd for each resolution and demanded minimum yield}

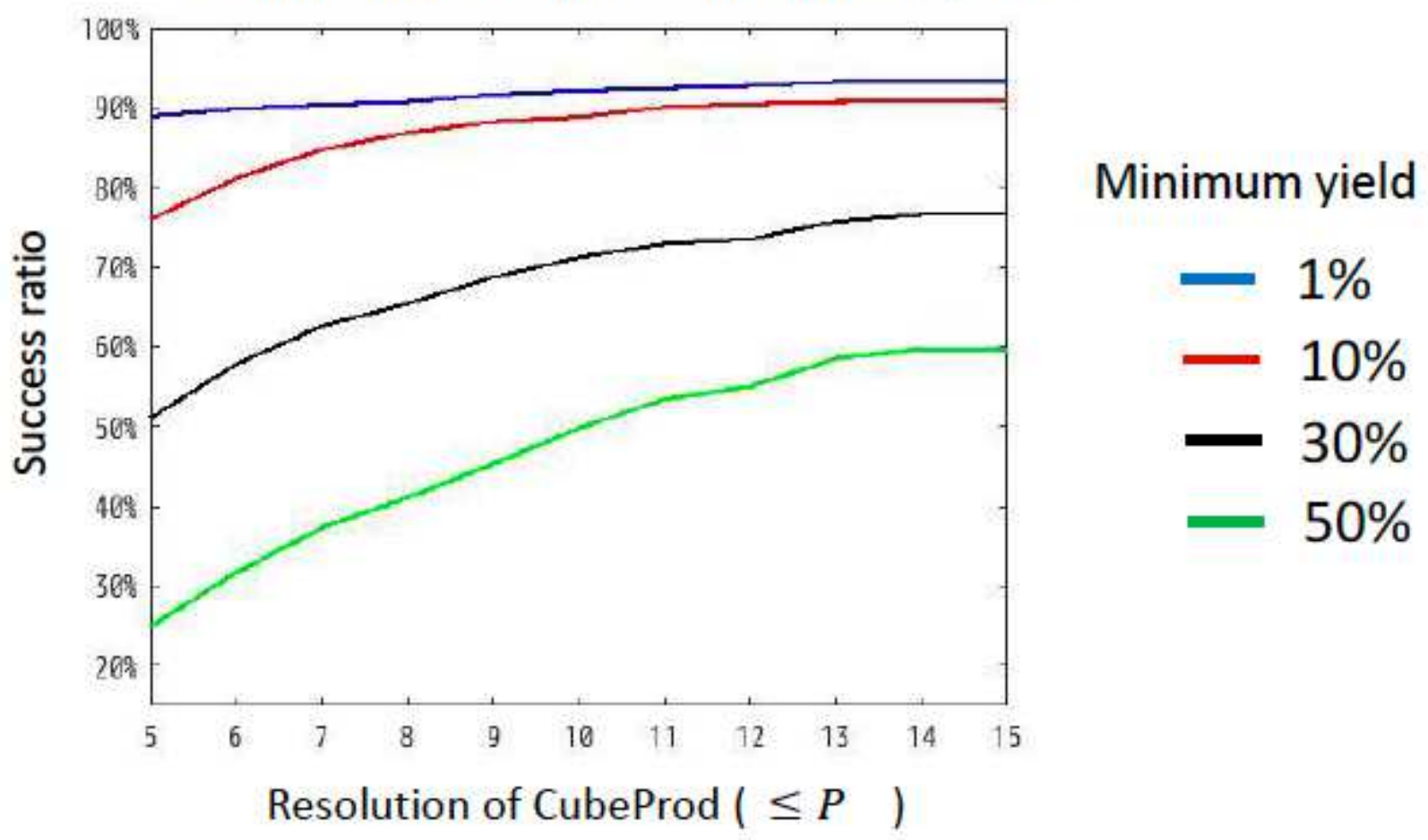

Figure 2

The success ratio of CubeProd in identifying reaction deletion strategies that could achieve growth coupling for 687 target metabolites of iMM904 under anaerobic conditions at each resolution $(\leq P)$ and demanded minimum yield. 


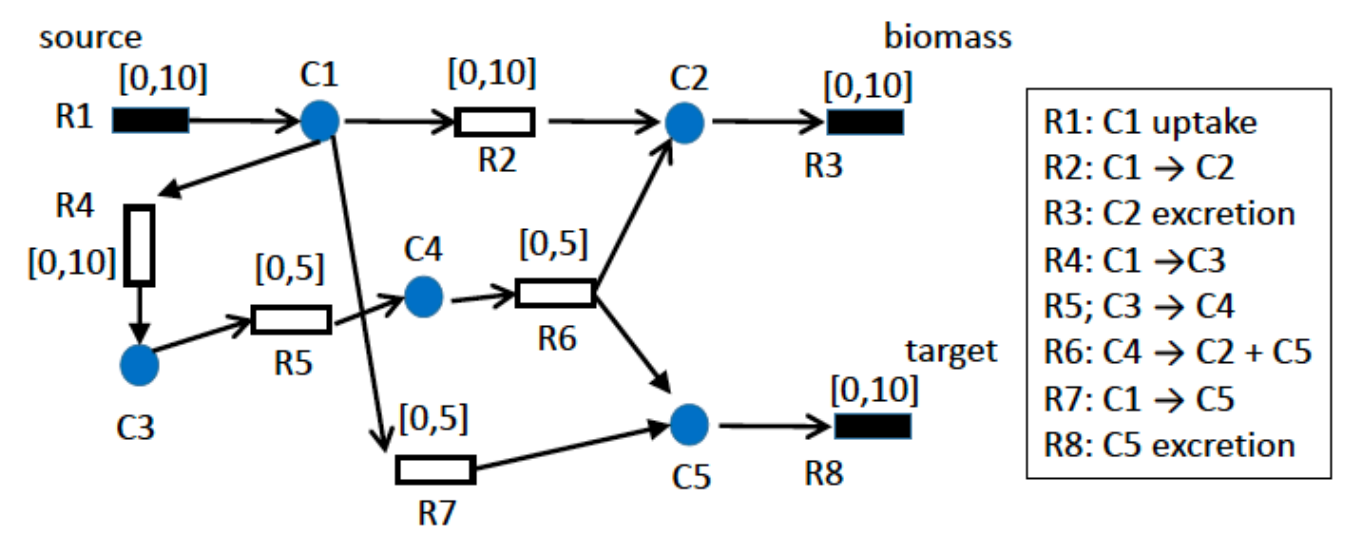

(A)

\begin{tabular}{|l|cccccccc|}
\hline Deletion & $\mathrm{R} 1$ & $\mathrm{R} 2$ & $\mathrm{R} 3$ & $\mathrm{R} 4$ & $\mathrm{R} 5$ & $\mathrm{R} 6$ & $\mathrm{R} 7$ & $\mathrm{R} 8$ \\
\hline none & 10 & $10-x_{6}$ & 10 & $x_{6}$ & $x_{6}$ & $x_{6}$ & 0 & 0 \\
$\mathrm{R} 2$ & 5 & 0 & 5 & 5 & 5 & 5 & 0 & 5 \\
$\mathrm{R} 2, \mathrm{R} 7$ & 5 & 0 & 5 & 5 & 5 & 5 & 0 & 5 \\
$\mathrm{R} 2, \mathrm{R} 6$ & 0 & 0 & 0 & 0 & 0 & 0 & 0 & 0 \\
\hline
\end{tabular}

(B)

\begin{tabular}{|c|c|lcccccccc|}
\hline $\mathrm{GR}$ & $\mathrm{PR}$ & & $\mathrm{R} 1$ & $\mathrm{R} 2$ & $\mathrm{R} 3$ & $\mathrm{R} 4$ & $\mathrm{R} 5$ & $\mathrm{R} 6$ & $\mathrm{R} 7$ & $\mathrm{R} 8$ \\
\hline $4 \leq \mathrm{GR} \leq 6$ & $4 \leq \mathrm{PR} \leq 6$ & 1st LP & 4 & 4 & 4 & 0 & 0 & 0 & 4 & 4 \\
& & 2nd LP & 10 & 10 & 10 & 0 & 0 & 0 & 0 & 0 \\
\hline $5 \leq \mathrm{GR} \leq 5$ & \multirow{2}{*}{$5 \leq \mathrm{PR} \leq 5$} & 1st LP & 5 & $\mathbf{5}$ & 5 & 0 & 0 & 0 & 5 & 5 \\
& & 2nd LP & 10 & 10 & 10 & 0 & 0 & 0 & 0 & 0 \\
\hline
\end{tabular}

(C)

\begin{tabular}{|c|c|c|lcccccccc|}
\hline $\mathrm{GR}$ & $\mathrm{PR}$ & $\mathrm{TAF}$ & & $\mathrm{R} 1$ & $\mathrm{R} 2$ & $\mathrm{R} 3$ & $\mathrm{R} 4$ & $\mathrm{R} 5$ & $\mathrm{R} 6$ & $\mathrm{R} 7$ & $\mathrm{R} 8$ \\
\hline $5 \leq \mathrm{GR} \leq 5$ & $5 \leq \mathrm{PR} \leq 5$ & $15 \leq \mathrm{TAF} \leq 15$ & $\mathrm{LP}$ & 5 & 0 & 5 & $\mathbf{5}$ & $\mathbf{5}$ & $\mathbf{5}$ & 0 & 5 \\
& & & FVA & 5 & 0 & 5 & 5 & 5 & 5 & 0 & 5 \\
\hline $4 \leq \mathrm{GR} \leq 6$ & $4 \leq \mathrm{PR} \leq 6$ & $12 \leq \mathrm{TAF} \leq 18$ & LP & 6 & 6 & 6 & 0 & 0 & 0 & $\mathbf{6}$ & 6 \\
& & & FVA & 10 & 10 & 10 & 0 & 0 & 0 & 0 & 0 \\
\hline $4 \leq \mathrm{GR} \leq 6$ & $4 \leq \mathrm{PR} \leq 6$ & \multirow{2}{*}{$12 \leq \mathrm{TAF} \leq 18$} & LP & 0 & 0 & 0 & $\mathbf{4}$ & $\mathbf{4}$ & $\mathbf{4}$ & 0 & 0 \\
& & & FVA & 10 & 10 & 10 & 0 & 0 & 0 & 0 & 0 \\
\hline
\end{tabular}

(D)

\section{Figure 3}

(A)A toy example in which CubeProd can identify the optimal strategy, but GridProd cannot. (B)The flux vectors for reaction deletions obtained by FVA minimizing R8 under the condition that R3 is maximized. (C)GridProd cannot find appropriate reaction deletions. (D)CubeProd can find appropriate reaction deletions if the resolution is large enough (cubes are small enough). 


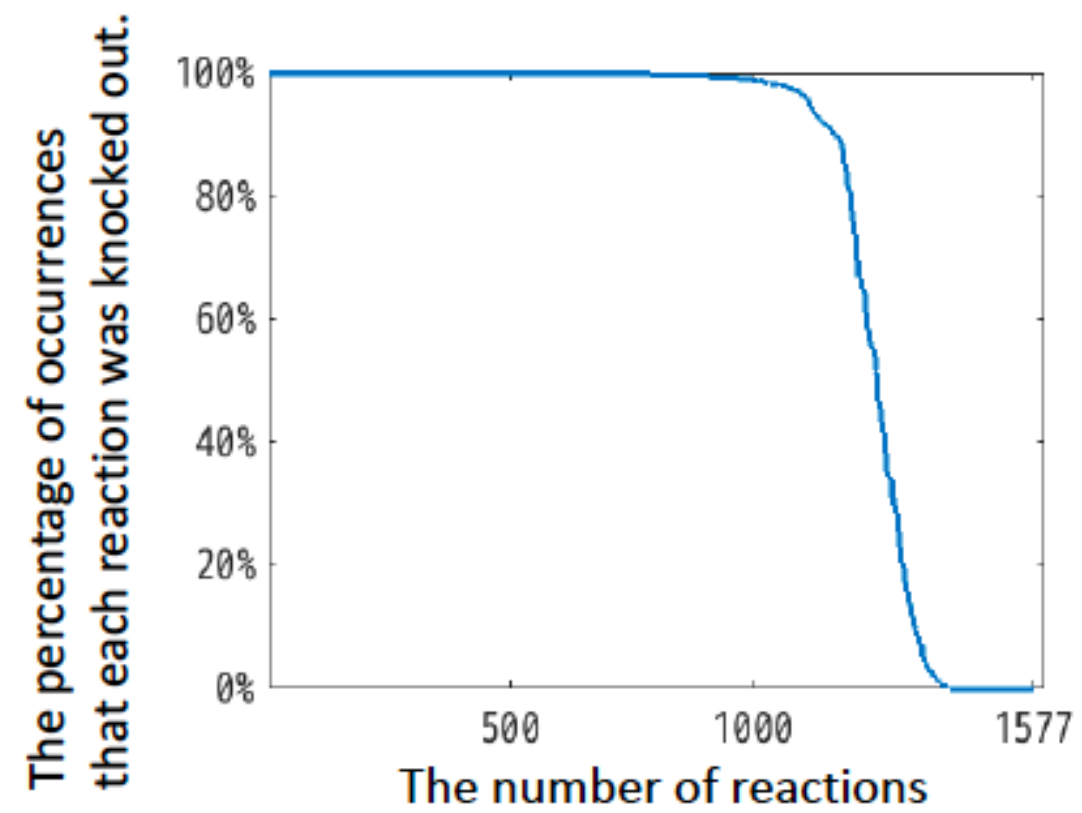

Figure 4

The percentage of occurrences each reaction was deleted in the Cube- Prod strategy.

\section{Supplementary Files}

This is a list of supplementary files associated with this preprint. Click to download.

- sourceCode.zip

- iMM904anaerobic.zip

- iJ01366anaerobic.zip

- iJ01366aerobic.zip

- yeast780anaerobic.zip

- yeast780aerobic.zip

- BTconstraintSearch.m

- README.txt

- CubeProd.m

- integrate.m

- targetMetCA2.mat

- example.m

- output.csv

- iMM904.mat 